\title{
VOLATILIDAD ESTOCÁSTICA, TEORÍA DE VALORES EXTREMOS Y VALUACIÓN DE DERIVADOS: CALIBRACIÓN Y ANÁLISIS DE 3 MODELOS DE PROCESOS ESTOCÁSTICOS PARA EL ÍNDICE DE LA BMV DE 1990 a 2005
}

\section{Andoni Gárritz Cruz*}

\author{
a.Sc Consultores, S.C.
}

(Recibido 10 de enero 2006, aceptado 24 de marzo 2006)

\begin{abstract}
In this paper three models of stochastic processes are tested both in terms of econometric fit and of their predictive power for the prices for derivatives traded in the Mexican derivatives market (MexDer). The asset studied is the Mexican Stock Exchange Index. The model proposed in the paper includes Poisson jumps, truncated Fréchet distributions and coherent measures of risk, as well as stochastic volatility. This model shows the best econometric fit and also the best predictive power, specially in 2006, when Puts are much more expensive than Black Scholes or a stochastic volatility CIR model predict. This paper has very important implications for compensation chambers and in the risk managemer.t of portafolios similar in structure to the index of the Mexican stock market.
\end{abstract}

\section{Resumen}

En este trabajo se calibra y se compara el desempeño de una familia de 3 modelos de procesos estocásticos con el fin de valuar un call y un put europeos sobre el índice de la Bolsa Mexicana de Valores (BMV). Los modelos van desde el clásico modelo de Merton-Black-Scholes hasta modelos con volatilidad estocástica y procesos de difusión estilo Poisson, cuyos saltos tienen distribución tipo Fréchet truncada. Se concluye que un modelo con volatilidad estocástica estilo CIR (que asegura que la varianza sea siempre positiva) junto con un proceso estocástico para el subyacente que incluye procesos estocásticos de Poisson con saltos cuya distribución es tipo Fréchet (saltos tanto hacia arriba como hacia abajo, y con la posibilidad de ser asimétricos) mostrará ser un modelo que permite explicar comportamientos múltiples para el volatility-smile tanto para un call como de un put europeos. Las distribuciones de Fréchet-Weibull se asocian a eventos extremos. Por ello, en este trabajo se utilizan dichas distribuciones para calibrar los modelos en los que hay procesos estocásticos tipo Poisson con el fin de explicar de manera más consistente el comportamiento de los precios de mercado observados en el período 1990-2006, particularmente en momentos de alta volatilidad. Las implicaciones de este trabajo son múltiples para fines de estimación de márgenes y primas en cámaras de compensación de derivados, así como para la estimación y administración de riesgos en portafolios similares al índice de la BMV.

Clasificación JEL : C1, G12

Palabras clave: Volatilidad estocástica, Teoría de Valores extremos, Derivados

* Calle 5 de mayo, no. 117, C. P. 16020, México D.F. Teléfono: +52(55) 30944446. Correo electrónico: agarritz@asc-consultores.com

El presente artículo se basa en el trabajo de tesis para obtener el grado de doctor en ciencias financieras por el Tecnológico de Monterrey, Campus Ciudad de México. 


\section{Introducción}

Existe desde hace décadas un gran interés en modelos de procesos estocásticos que puedan describir el comportamiento de los precios de varios activos financieros. Los procesos estocásticos que son martingalas son de particular interés para la valuación de derivados.

En un inicio se intentó describir a los precios de los activos mediante el supuesto de que los log-rendimientos tienen distribución normal. Esto implica que es posible encontrar los parámetros relevantes mediante regresiones por mínimos cuadrados o maximización de la función de verosimilitud. Eventualmente, mediante el ajuste y análisis econométrico de múltiples series de tiempo de activos financieros, se observó que en la mayoría de los casos los residuales de dichas regresiones mostraban muchos indicios de no ser normales. Debido a que se viola el supuesto de los modelos de regresión en los que se asume que los residuales de las regresiones son normales, entonces se han propuesto otro tipo de modelos, entre ellos el propuesto por Bollerslev (1986): el modelo GARCH.

En dicho modelo se asume que la volatilidad es estocástica, y que muestra reversión a la media. En GARCH se hace una estimación conjunta de los parámetros de dos ecuaciones simultáneas: 1) la ecuación de los logrendimientos y 2) la ecuación de la volatilidad. Por un lado, existe evidencia en múltiples análisis y publicaciones de que los residuales de regresiones tipo GARCH aplicados a series de tiempo de alta frecuencia aún muestran exceso de kurtosis, sesgo y además colas asimétricas. Esto implica que los procesos GARCH son aún insuficientes para modelar correctamente los procesos estocásticos, pues uno de los supuestos en las regresiones tipo GARCH es que los residuales de ambas regresiones son normales. Este supuesto se viola la mayoría de las veces en las series de tiempo de múltiples activos financieros. Esto se ha intentado corregir con otro tipo de regresiones, como las tipo TARCH y EGARCH, que permiten asimetría en la distribución de probabilidad. Sin embargo, estos modelos siguen fallando en lo referente a la normalidad de los residuales. En este trabajo se propone un método de medición econométrica distinto al de regresión tradicional tipo TARCH o EGARCH.

Por otro lado, se ha tratado de explicar el precio de un derivado mediante distintas propuestas de procesos estocásticos que son martingalas. Black y Scholes asumen que la distribución de probabilidad de los log-rendimientos infinitesimales de un activo es normal con media $\left(\mu-\frac{1}{2} \sigma^{2}\right) d t$ y volatilidad $\sigma \sqrt{d t}$. Esto implica que la fdp del precio del subyacente en el futuro se distribuye como lognormal. Este supuesto en el modelo de Black-Scholes no puede explicar correctamente los precios de mercado de derivados. En específico, B-S no puede explicar las volatilidades implícitas que se observan en el mercado. Esto se debe primordialmente a que, en la realidad, la fdp de los logrendimientos infinitesimales no es normal. Para corregir este problema se han propuesto modelos con volatilidad estocástica (entre ellos los tipo GARCH), modelos con saltos deterministas, modelos con saltos estocásticos y mezclas de éstos. Nelson (1990) muestra la relación entre los procesos GARCH y los procesos de difusión con saltos. Se advierte que un proceso de difusión con saltos provoca sesgos en las estimaciones GARCH y genera residuales no normales. Bates (1996), por ejemplo, muestra que para el tipo de cambio marco alemán-dólar el proceso que mejor describe los precios de mercado de derivados entre 5 modelos de procesos 
estocásticos distintos es una mezcla de un proceso browniano con volatilidad estocástica con ecuación funcional tipo Cox-Ingersol-Ross (CIR), más un proceso de Poisson para describir los saltos, y asume que sólo hay saltos hacia arriba y que éstos se distribuyen como una lognormal. Estos supuestos producen colas anchas y asimétricas debido tanto a la volatilidad estocástica como al proceso de Poisson.

En la mayoría de los artículos publicados sobre procesos estocásticos con saltos de Poisson suele asumirse adicionalmente que la distribución de probabilidad de la magnitud de los saltos es de tipo lognormal. Este supuesto hace posible encontrar soluciones cerradas para los precios de ciertos derivados bajo estos procesos estocásticos, y suelen implicar sumatorias infinitas de Calls (o Puts) a la Black-Scholes (ver Merton, 1996). En este trabajo no se asumirá que los saltos son únicamente hacia arriba, y con distribución lognormal (tal y como propone Bates en 1996 para explicar colas anchas y asimétricas para la distribución de los log-rendimientos), sino que hay saltos tanto hacia arriba como hacia abajo, y cuyas distribuciones de probabilidad pertenecen a la familia de distribuciones de probabilidad derivadas de la teoría de valores extremos. Además, el modelo propuesto incluye también volatilidad estocástica. Esto se hace con la finalidad de unir a las teorías de valuación de derivados, de la volatilidad estocástica, de valores extremos, de martingalas equivalentes y de medidas coherentes de riesgo.

Se compararán además los precios teóricos que predicen los 3 modelos de procesos estocásticos con los precios observados en la realidad en el mercado de derivados sobre el indice de la BMV, y se explorará también a los modelos desde el punto de vista de parsimonia, bondad de ajuste y pruebas de normalidad para los residuales. El problema que se tiene para hacer el ajuste a los precios de mercado es que el mercado de derivados en México comenzó a operar apenas en marzo del 2004, y sólo se han emitido 3 familias de derivados (marzo, junio, septiembre de 2004) hasta la fecha en la que se realizó dicha búsqueda. En 2004-2006 se vive un ambiente de volatilidad baja, así como de tasas de interés relativamente bajas y estables. Es posible que esto no se mantenga así en el futuro y, por ello, el análisis de esta trabajo desde el punto de vista de ajuste a las observaciones de precios de mercado será limitado debido a la falta de datos.

\subsection{Marco Teórico}

Existen teorías ideadas por los economistas "behaviouralists" que pueden explicar el hecho de que los rendimientos no sean normales. En el libro de Neil Johnson ("Financial Market Complexity", 2003) se detalla cómo modelar una economía partiendo del supuesto de que existen muchos individuos distintos que interactúan entre sí en un mercado virtual modelado y simulado en una computadora. En particular hay evidencia de que el agente promedio en el mercado difiere en mercados alcistas y en mercados bajistas, tal y como se puede inferir del artículo de Kim, M. y Ismail, B. (1998). Esto explica la asimetría de la distribución de probabilidad de los log-rendimientos $\mathrm{y}$, por lo tanto, que la aversión al riesgo del inversionista "promedio" difiere en mercados alcistas (bull) o mercados bajistas (bear).

Por otro lado, Bates (1996), Braun (1995) y Engle (1993) han mostrado que las noticias no esperadas por el mercado (buenas o malas) producelı saltos en los rendimientos que inducen no-normalidad en las distribuciones de probabilidad 
de los logrendimientos. En este trabajo el enfoque es similar al propuesto por estos autores, pues se toman 3 distribuciones: una normal para explicar a la mayoría de los eventos, y dos distribuciones Fréchet truncadas para explicar los eventos extremos. Este enfoque, coherente desde el punto de vista de medición de riesgos, hace uso de teoremas de la teoría de valores extremos (en particular la condición de Von Mises, el teorema de Fisher-Tippet y otros teoremas) para justificar el uso de la distribución Fréchet.

\subsection{Teoría de valores extremos}

Esta teoría se ocupa de explicar desde el punto de vista teórico el uso de ciertas familias de funciones de distribución de probabilidad para describir los eventos extremos de variables aleatorias con cualquier distribución de probabilidad, y que cumpla con ciertas condiciones. En el libro de Embrechts, Klüppelberg y Mikosch se detalla la teoría de valores extremos. En particular, se detalla la justificación del uso de la función de Fréchet para modelar eventos extremos (entre varias otras funciones).

En la parametrización Alfa (donde $\alpha=\frac{1}{\gamma}$ ), la función de Fréchet es:

$$
G_{1}(x ; \alpha, \mu, \sigma)=e^{-\left(\frac{x-\mu}{\sigma}\right)^{-\alpha}}
$$

Para el caso particular de valores de gama infinitos (o valores de Alfa iguales a cero) se tiene entonces la función de Gumbell:

$$
G_{0}(x)=e^{-e^{-\left(\frac{x-\mu}{\sigma}\right)}}
$$

La pregunta es ahora qué se va a definir como "máximos". Para poder hacerlo habrá que ordenar las observaciones que se tengan de la mayor a la menor y quedarnos con cierto número " $k$ " de máximos observados, y entonces encontrar los parámetros de la Fréchet que mejor describen a la cola de la distribución. El número " $k$ " es entonces una variable que se debe definir. Para resolver este problema se acude a lo siguiente:

\subsubsection{Estabilidad del máximo}

F tiene estabilidad del máximo si existen sucesiones $a_{n}$ y $b_{n}$ tales que

$$
F^{n}\left(b_{n}+a_{n} x\right)=F(x)
$$

Para las funciones EV0, EV1 y EV2, se tiene que

$$
\text { (Para la EV0: } b_{n}=\log n, a_{n}=1 \text { ) }
$$

(Para la EV1 y EV2: $b_{n}=0, a_{n}=\frac{1}{\alpha}$ ) 


\subsubsection{Dominio de atracción}

Una de las consecuencias de lo descrito anteriormente es que una muestra de variables aleatorias que sean los "máximos" de una muestra tenderá a distribuirse como Fréchet si se cumple la condición de Von Mises para el dominio de atracción de la función de Fréchet:

$$
\begin{gathered}
\lim _{x \rightarrow \infty} \frac{x f(x)}{\tilde{F}(x)}=\alpha>0 \\
\tilde{F}(x)=1-F(x)
\end{gathered}
$$

En otras palabras, hay que escoger un número de máximos que caiga en una zona en donde los valores de Alfa (o Gama) sean estables. Si se escoge un umbral demasiado alto, el número de máximos será escaso, y consecuentemente la cofiabilidad de la estimación baja. Si se toma un umbral demasiado bajo se estarán incorporando observaciones "no extremas" a las verdaderamete "extremas", contaminando así los resultados de las estimaciones. Los paquetes de software estadístico (como el X-tremes) obtienen fácilmente diagramas donde se muestra el valor obtenido de $\alpha$ para distintos números de máximos escogidos. Debe escogerse entonces un número de máximos que esté en una zona en la que $\alpha$ es estable.

\subsubsection{Medidas coherentes de riesgo}

En la literatura se ha discutido sobre el concepto de medidas coherentes de riesgo desde hace ya tiempo. Para Markowitz (1952) el riesgo se puede conceptualizar como la varianza. A mayor varianza, mayor riesgo. De este supuesto de derivan varias teorias de valuación de activos, como el CAPM. El problema con esta medida del riesgo es que no es coherente pues asume, entre otras cosas, que la distribución de probabilidad de los rendimientos de los activos financieros es normal multivariado, e invariante en el tiempo. Otros defectos de este enfoque se mencionan posteriormente. Otro modelo popular de medición del riesgo es el Value-at-Risk (VaR). Se ha criticado al VaR por el hecho de no ser subaditivo (a menos que se utilicen modelos multivariados).

Acorde con Artzner ("Coherent Measures of Risk", 1999), las medidas coherentes de riesgo deben de cumplir con las siguientes características:

Sea $\rho(x)$ una medida de riesgo de $\mathrm{x}$ (las pérdidas posibles, no confundir con el coeficiente de correlación). Entonces, $\rho(x)$ debe cumplir con:

1) Subaditividad: $\rho(x+y) \leq \rho(x)+\rho(y)$. Es decir, que diversificar disminuye el riesgo.

2) Monotonicidad: $\mathrm{Si} x \leq y$, entonces $\rho(x) \leq \rho(y)$.

3) Homogeneidad positiva: $\forall \lambda \geq 0, \rho(\lambda x)=\lambda \rho(x)$.

4) Invarianza bajo translaciones: Para alguna constante $\alpha, \rho(x+\alpha)=\rho(x)+\alpha$. Un posible ejemplo de una medida coherente de riesgo que cumple con los 4 principios es:

$$
\rho(x)=\int_{V a R_{\gamma \%}}^{\infty} x f(x) d x
$$


Donde $f(x)$ es la función de distribución de probabilidad de las pérdidas. En otras palabras, el valor que nos interesa es la pérdida esperada dado que se excede al VaR (o un umbral cualquiera), y no el VaR en sí mismo.

En particular, en esta trabajo se estudia el caso de la función de distribución de Fréchet para estimar esta medida coherente de riesgo. La función de Fréchet funciona bien para describir los máximos de alguna muestra. Los umbrales que separan a los "máximos" de los "no-máximos" son los que corresponderían al VaR al $97.5 \%$ de certeza estadística, asumiendo que los logrendimientos son normales. La siguiente gráfica muestra los umbrales que se obtuvieron:

\section{Gréfica 1.}

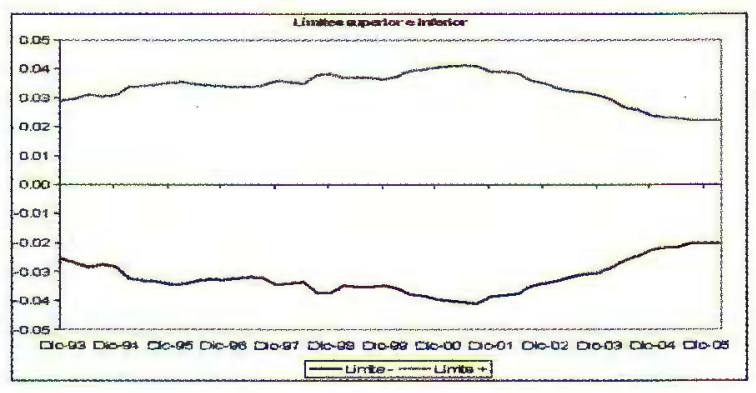

Sea u un umbral definido por la media $+/-1.96$ veces la desviación estándard de los logrendimientos diarios (tal y como se observa en la gráfica arriba). Tenemos entonces a dos umbrales $u^{-}$y $u^{+}$, que definirán los límites a partir de los cuales se valuarán las colas de la distribución. Se observa que $u^{-}$y $u^{+}$representan el $V a R_{97.5 \%}$ (asumiendo Gaussianidad) para una posición corta y para una posición larga, respectivamente. Una vez definido este umbral se filtrarán las series para obtener la serie de tiempo de los máximos, de los mínimos y de las observaciones restantes, que representan el movimiento browniano. Una vez filtrados los datos se procederá a ajustar las funciones de Fréchet que mejor describan a los eventos extremos. Una vez encontrados los parámetros de la función de Fréchet se puede encontrar fácilmente el VaR al 97.5\% utilizando la ventaja de que $\mathrm{G}$ tiene una función de cuantiles sencilla: $\sigma_{f}$ es:

Sea $q \in[0,1]$. La función de cuantiles de Fréchet para parámetros $\alpha, \mu_{f}$ y

$$
G^{-1}(q)=(-\ln (q))^{-\frac{1}{\alpha}}
$$

De lo que se deriva que, si $q=0.975$, entonces:

$$
\text { VaR } R_{97.5 \% \text { Frchet }}=\mu_{f}+\sigma_{f}(-\ln (0.975))^{-\frac{1}{\alpha}}
$$

Sea u un umbral cualquiera. La función de Fréchet hasta ese umbral es: 


$$
G\left(u ; \alpha, \mu_{f}, \sigma_{f}\right)=e^{-\left(\frac{u-\mu_{f}}{\sigma_{f}}\right)^{-\alpha}}
$$

Esta es la probabilidad de que se obtenga un brinco menor que el umbral preestablecido. Para normalizar a 1 la función de distribución de probabilidad desde el umbral al infinito simplemente se divide entre 1-G(u). Una vez normalizada a 1 se puede encontrar la fdp de los excedentes al umbral u y, por ende, poder estimar el valor esperado de las pérdidas dado que se excedió un umbral. Se propone entonces la siguiente medida coherente de riesgo:

$$
\rho(x)=\frac{1}{1-G\left(u ; \alpha, \mu_{f}, \sigma_{f}\right)} \int_{u}^{\infty} x g(x) d x
$$

Se observa que esto no es más que:

$$
\rho(x)=E[x \mid x>u]
$$

Donde $\mathrm{x}$ tiene distribución Fréchet truncada con límite inferior igual a u. Hay que recordar que u (el VaR al 97.5\%) se refiere a la distribución original, y asume Gaussianidad. No hay que confundir este VaR al $97.5 \%$ con el verdadero VaR al 97.5\% (que debe calcularse con la distribución de Fréchet y no con la normal). La manera correcta de calcular la pérdida esperada dado que se excedió un umbral consiste en estimar con un umbral igual al $V a R_{97.5 \% \text { Frechet }}$. En ese caso, $G\left(u, \alpha, \mu_{f}, \sigma_{f}\right)=0.975$. La medida coherente de riesgo es entonces:

$$
\rho(x)=40 \int_{\text {VaR } 97.5 \% \text { Frechet }}^{\infty} x g(x) d x
$$

El problema con esta medida es que es inconsistente con la medición econométrica que se hizo en este trabajo. En la medición econométrica se filtraron las series de tiempo y se asumió que todas las observaciones con valores mayores al VaR Gaussiano serían asignadas como "máximos" (un porcentaje de dichas observaciones). Para ser consistentes entre la medición econométrica y la simulación Montecarlo, la medida de riesgo debe ser la propuesta previamente:

$$
\begin{gathered}
\rho(x)=\frac{1}{1-G(u ; \alpha, \mu, \sigma)} \int_{u}^{\infty} x g(x) d x \\
u=\operatorname{VaR}_{97.5 \% \text { Gaussiano }}
\end{gathered}
$$

Esta pérdida esperada $\rho(x)$ será estimada de manera numérica. Si se conocen los parámetros de la distribución Fréchet se puede estimar eficientemente la pérdida esperada dado que se excede el umbral mediante métodos numéricos de integración. 


\subsection{Modelo CIR para Volatilidad Estocástica y su relación con el modelo $\operatorname{GARCH}(1,1)$}

Debido a las propiedades de la distribución de probabilidad del modelo CIR en el sentido de que no es posible obtener valores negativos para el subyacente, se puede utilizar un modelo CIR para modelar la volatilidad estocástica (el comportamiento de la volatilidad es ahora similar al proceso estocástico antes propuesto para la tasa de interés):

$$
\begin{gathered}
d S=\mu S d t+\sigma_{w} S d W_{t} \\
d \sigma_{w}^{2}=a\left(b-\sigma_{w}^{2}\right) d t+\theta \sigma_{w} d Z_{t} \\
\operatorname{COV}\left(d W_{t}, d Z_{t}\right)=\rho d t
\end{gathered}
$$

Cox-Ingersoll-Ross encuentran una fórmula cerrada para corregir el precio de opciones europeas. Encuentran que, por lo general, los precios obtenidos son más altos que los obtenidos por Black-Scholes para plazos largos. Las únicas excepciones son opciones con maduración muy corta y que comiencen con un valor muy bajo de volatilidad.

En el caso en el que la correlación $\rho$ es cero, entonces el modelo CIR de volatilidad estocástica es muy parecido, mas no igual, a otro modelo muy utilizado: el GARCH(1,1). Willmott (en "Paul Willmott on Quantitative Finance", 2000) menciona que el proceso de estimación $\operatorname{GARCH}(1,1)$ desde el punto de vista econométrico consiste en un par de ecuaciones que definen al subyacente y a la volatilidad que es muy similar al propuesto aquí, y las diferencias son: 1) en la ecuación de la volatilidad la parte estocástica en $\operatorname{GARCH}(1,1)$ depende de $\sigma_{w}^{2}$, mientras que en el modelo propuesto depende de la volatilidad, y no del cuadrado de la misma. Esta diferencia afecta sólo a la parte estocástica (es decir, a los residuales de la segunda ecuación), y no afecta en el sentido de que la parte determinista es idéntica en ambos modelos. Es decir: asumen ambos que la volatilidad tiende en el largo plazo al valor "b" a una velocidad " $a$ "; y 2) GARCH $(1,1)$ asume que la covarianza entre $d W_{t}$ y $d Z_{t}$ es cero, mientras que el modelo propuesto permite que dicha variable sea distinta de cero.

Haciendo la sustitución de variables $x=2 \sigma$ obtenemos la ecuación que rige el comportamiento de la volatilidad:

$$
\sigma_{t+d t}=\beta_{0} \frac{1}{\sigma_{t}}+\beta_{1} \sigma_{t}+\theta \sqrt{\Delta t} \epsilon
$$

Donde

$$
\begin{aligned}
& \epsilon \sim N(0,1) \\
& \beta_{0}=\frac{a b}{2}-\frac{\theta^{2}}{8} \\
& \beta_{1}=1-\frac{a}{2}
\end{aligned}
$$




\section{Los Modelos}

Modelo con saltos tipo Poisson-Fréchet asimétricos hacia arriba y hacia abajo, con volatilidad estocástica y con correlación entre el movimiento browniano del subyacente y el movimiento browniano de la volatilidad.

En los modelos que se analizan en este trabajo los primeros 2 modelos son casos particulares del caso 3 . Los modelos 1 y 2 son casos restringidos del modelo 3 , en donde se fuerza a que algunos de los coeficientes sean cero. Por esta razón, se analizará a detalle el desarrollo teórico que sustenta al modelo 3 , pues éste sustentará también a los demás 2.

El proceso estocástico que se analiza es el siguiente:

$$
\begin{gathered}
d S=\mu S d t+\sigma_{w} S d W_{t}+\left(e^{\nu^{+}}-1\right) S d N_{t}^{+}+\left(e^{-\nu^{-}}-1\right) S d N_{t}^{-} \\
d \sigma_{w}^{2}=a\left(b-\sigma_{w}^{2}\right) d t+\theta \sigma_{w} d Z_{t} \\
\operatorname{COV}\left(d W_{t}, d Z_{t}\right)=\rho d t
\end{gathered}
$$

$d W_{t}$ y $d Z_{t}$ son procesos brownianos estándard sin drift y correlación $\rho$. $d N_{t}^{+}$es un proceso de difusión tipo Poisson con factor de intensidad $\lambda^{+}$. $d N_{t}^{-}$es un proceso de difusión tipo Poisson con factor de intensidad $\lambda^{-}$. $\nu^{+}$se distribuye como una Fréchet truncada con parámetros $\mu^{+}, \sigma^{+}, \alpha^{+}$, y con umbral $u^{+}$.

$\nu^{-}$se distribuye como una Fréchet truncada con parámetros $\mu^{-}, \sigma^{-}, \alpha^{-}$, y con umbral $u^{-}$

Y se sabe además que $\nu^{+}$y $\nu^{-}$sólo muestran valores excedentes a un umbral predefinido (deben exceder a la media en más de 1.96 desviaciones estándard). En otras palabras, sólo nos quedaremos con un trozo de la distribución de Fréchet: aquel trozo en donde se excede la media de los logrendimientos en 1.96 veces la desviación estándard de los logrendimientos. Para lograr una cobertura (hedge) más o menos perfecta debemos hacer uso del valor de la medida coherente de riesgo $\rho(x)$ para lograr una valuación adecuada de los derivados.

Cualquier función del par de ecuaciones descritas arriba cumple con el cálculo de Itô generalizado para incorporar brincos:

Sea $f=f\left(S, \sigma_{w}^{2}, t\right)$. Entonces

$$
\begin{gathered}
d f \approx \frac{\partial f}{\partial t} d t+\frac{\partial f}{\partial S} d S+\frac{\partial f}{\partial \sigma_{w}^{2}} d \sigma_{w}^{2}+ \\
+\frac{1}{2} \frac{\partial^{2} f}{\partial S^{2}} d S^{2}+\frac{1}{2} \frac{\partial^{2} f}{\partial\left(\sigma_{w}^{2}\right)^{2}} d\left(\sigma_{w}^{2}\right)^{2}-\frac{\partial^{2} f}{\partial S \partial \sigma_{w}^{2}} d S d \sigma_{w}^{2}+ \\
+\left[f\left(S\left(1+e^{\nu^{+}}-1\right), t\right)-f(S, t)\right] d N^{+}+\left[f\left(S\left(1+e^{-\nu^{-}}-1\right), t\right)-f(S, t)\right] d N^{-}
\end{gathered}
$$


Para el caso específico $f\left(S, \sigma_{w}^{2}, t\right)=\ln S$ tendremos que:

$$
d(\ln S)=\left(\mu-\frac{1}{2} \sigma_{\text {tot }}^{2}\right) d t+\sigma_{w} d W+\nu^{+} d N^{+}-\nu^{-} d N^{-}
$$

Donde

$$
\sigma_{\text {tot }}^{2}=\sigma_{w}^{2}+\lambda^{+} d t E\left[\left(\nu^{-} \mid x>u^{-}\right)^{2}\right]+\lambda^{-} d t E\left[\left(\nu^{-} \mid x>u^{-}\right)^{2}\right]
$$

El proceso estocástico para $d(\ln S)$ en el mundo neutral al riesgo es entonces (x representa valores posibles de los logrendimientos):

$$
\begin{gathered}
d(\ln S)=\left(r-\frac{1}{2} \sigma_{\text {tot }}^{2}-\lambda^{+} E\left[\nu^{+} \mid x>u^{+}\right]+\lambda^{-} E\left[\nu^{-} \mid x>u^{-}\right]\right) d t \\
+\sigma_{w} d \tilde{W}+\left(\nu^{+} \mid x>u^{+}\right) d N^{+}-\left(\nu^{-} \mid x>u^{-}\right) d N^{-} \\
d \sigma_{w}^{2}=a\left(b-\sigma_{w}^{2}\right) d t+\theta \sigma_{w} d Z_{t} \\
\operatorname{COV}\left(d \tilde{W}_{t}, d Z_{t}\right)=\rho d t
\end{gathered}
$$

En las ecuaciones anteriores $\sigma_{\text {tot }}$ es la volatilidad de todo el proceso estocástico. Dado que se asume que $d W, d N^{+}$y $d N^{-}$son independientes entonces

$$
\sigma_{\text {tot }}^{2}=\sigma_{w}^{2}+\lambda^{+} d t E\left[\left(\nu^{-} \mid x>u^{-}\right)^{2}\right]+\lambda^{-} d t E\left[\left(\nu^{-} \mid x>u^{-}\right)^{2}\right]
$$

En la ecuación anterior las esperanzas se obtienen mediante integración numérica utilizando los parámetros de la Fréchet truncada. $d \tilde{W}$ es un proceso browniano sin drift y $\sigma_{w}^{2}$ sigue el proceso estocástico tipo CIR descrito anteriormente. Se observa que, dado que $E\left[\nu^{+} \mid x>u^{+}\right]=\rho\left(\nu^{+}\right)$y $E\left[\nu^{-} \mid x>u^{-}\right]=\rho\left(\nu^{-}\right)$, entonces:

$$
\begin{aligned}
& d(\ln S)=\left(r-\frac{1}{2} \sigma_{\text {tot }}^{2}-\lambda^{+} \rho\left(\nu^{+}\right)+\lambda^{-} \rho\left(\nu^{-}\right)\right) d t+ \\
& +\sigma_{w} d \tilde{W}+\left(\nu^{+} \mid x>u^{+}\right) d N^{+}-\left(\nu^{-} \mid x>u^{-}\right) d N^{-}
\end{aligned}
$$

Sea ahora $\tilde{S}=S e^{-r(T-t)}$, donde $\mathrm{T}$ es alguna fecha posterior a $t$.

$$
\begin{gathered}
d(\ln \tilde{S})=\left(-\frac{1}{2} \sigma_{\text {tot }}^{2}-\lambda^{+} \rho\left(\nu^{+}\right)+\lambda^{-} \rho\left(\nu^{-}\right)\right) d t+ \\
+\sigma_{w} d \tilde{W}+\left(\nu^{+} \mid x>u^{+}\right) d N^{+}-\left(\nu^{-} \mid x>u^{-}\right) d N^{-}
\end{gathered}
$$

$d \tilde{W}$ es un movimiento browniano estándard sin tendencia.

El proceso que sigue $d \tilde{S}$ en el mundo donde la cuenta bancaria es numeraria es entonces:

$$
\begin{gathered}
d \tilde{S}=\left(-\lambda^{+} \rho\left(\nu^{+}\right)+\lambda^{-} \rho\left(\nu^{-}\right)\right) \tilde{S} d t+ \\
+\sigma_{w} \tilde{S} d \tilde{W}+\left(e^{\nu^{+} \mid x>u^{+}}-1\right) \tilde{S} d N^{+}-\left(e^{\nu^{-} \mid x>u^{-}}-1\right) \tilde{S} d N^{-}
\end{gathered}
$$


Se observa que $d \tilde{S}$ es una martingala (recordar que $e^{x} \approx 1+x$ para $\mathrm{x}$ pequeñas). Cuando $\Delta t \rightarrow 0$ (es decir, cuando se cuente con una serie de tiempo con alta frecuencia) el tamaño de los saltos es muy pequeño y puede utilizarse esta aproximación. Dado que sucede lo arriba descrito, entonces debe pasar que si se hacen miles de corridas de la simulación Montecarlo con un $\Delta t$ pequeño, el valor de $e^{\nu \mid x>\imath}-1$ tiende a $\rho(\nu)$. Este proceso estocástico puede ser utilizado para hacer las simulaciones Montecarlo para valuar un Call o un Put Europeos (y también opciones americanas, bermudianas y otros derivados). Otro aspecto sobresaliente de esta simulación Montecarlo es que hay que introducir los valores estimados de las funciones de medidas coherentes de riesgos del subyacente en el lapso $\Delta t$ para poder valuar algún derivado.

\subsection{Valuación de un derivado}

La teoría de valuación mediante martingalas equivalentes menciona esencialmente que en un mundo en el que el subyacente es una martingala el valor de algún derivado cumple con

$$
D_{t}=e^{-r t} \tilde{E}\left[g(\tilde{S}) \mid \tilde{S}_{t}\right]
$$

Donde g(.) es alguna función de pago y $\tilde{E}$ es la esperanza medida en un mundo donde un bono sin riesgo a plazo $\mathrm{T}$ es la numeraria y en donde hay neutralidad al riesgo (es decir, en el mundo "martingalizado" en donde la distribución infinitesimal de rendimientos tiene esperanza cero). Además, se sabe que $\tilde{S}_{t}=S_{t}$. En particular, un call europeo toma la forma

$$
C_{t}=\tilde{E}\left[\max \left(\tilde{S}_{T}-K e^{-r(T-t)}, 0\right) \mid \tilde{S}_{t}\right]
$$

Y un Put europeo toma la forma

$$
P_{t}=\tilde{E}\left[\max \left(K e^{-r(T-t)}-\tilde{S}_{T}, 0\right) \mid \tilde{S}_{t}\right]
$$

El valor esperado dada la medida de probabilidad en donde $d \tilde{S}_{t}$ es martingala se encontrará mediante simulaciones Montecarlo que repliquen los 12 procesos estocásticos deseados para simular el precio futuro a un tiempo $T$ del precio del subyacente y su volatilidad, así como la intensidad y la ocurrencia de los saltos. Los detalles de dichas simulaciones se encuentran en secciones posteriores de este trabajo.

El libro de Musiela y Rutkowsky ("Martingale methods in financial modelling", 1998) es excelente para profundizar en los conocimientos sobre la valuación de derivados mediante el uso de martingalas equivalentes. Las martingalas equivalentes toman la distribución de probabilidad original y las modifican de manera tal que la distribución de rendimientos infinitesimales se convierte en una martingala.

Los modelos a analizar son los siguientes, ascendiendo en orden de complejidad: en todos los casos se asumirá que

$$
\left(\begin{array}{c}
d W \\
d Z
\end{array}\right) \sim N\left(\left(\begin{array}{l}
0 \\
0
\end{array}\right),\left(\begin{array}{ll}
1 & \rho \\
\rho & 1
\end{array}\right) d t\right)
$$




$$
\rho=\operatorname{CORRE} L(d W, d Z)
$$

Aún más general, además del supuesto de normalidad bivariada entre dW y dZ, lo que se asumirá es que el vector aleatorio expandido muestra la matriz de covarianza siguiente:

$$
\operatorname{COV}\left(\begin{array}{c}
d W \\
d Z \\
d N^{-} \\
d N^{+}
\end{array}\right)=\left(\begin{array}{cccc}
1 & \rho & 0 & 0 \\
\rho & 1 & 0 & 0 \\
0 & 0 & \lambda^{+} & 0 \\
0 & 0 & 0 & \lambda^{-}
\end{array}\right) d t
$$

Donde $\lambda^{+} \mathrm{y}^{-}$son los parámetros de intensidad del proceso de difusión de Poisson (recordar que la varianza de un proceso de Poisson es igual al factor de intensidad del mismo).

\section{Modelo 1:}

Este es el modelo clásico de Merton-Black-Scholes.

$$
d S=\mu S d t+\sigma_{w} S d W
$$

Asume volatilidad determinista y no hay procesos de difusión tipo Poisson.

Modelo 2:

Modelo con volatilidad estocástica estilo CIR y con correlación cero. Parecido a un modelo GARCH $(1,1)$.

$$
\begin{gathered}
d S=\mu S d t+\sigma_{w} S d W \\
d \sigma_{w}^{2}=a\left(b-\sigma_{w}^{2}\right) d t+\theta \sigma_{w} d Z \\
\operatorname{COV}(d W, d Z)=0
\end{gathered}
$$

Modelo 3:

Modelo con 2 procesos de difusión tipo Poisson y distribuciones Fréchet. Para los saltos y con volatilidad estocástica. Hay saltos tanto hacia arriba como hacia abajo, y pueden ser asimétricos. La correlación entre dW y dZ es igual a $\rho$.

$$
\begin{gathered}
d S=\mu S d t+\sigma_{w} S d W+\left(e^{\nu^{+}}-1\right) S d N^{+}+\left(e^{-\nu^{-}}-1\right) S d N^{-} \\
d \sigma_{w}^{2}=a\left(b-\sigma_{w}^{2}\right) d t+\theta \sigma_{w} d Z \\
\operatorname{COV}\left(d W, d N^{+}\right)=0 ; \operatorname{COV}\left(d W, d N^{-}\right)=0 ; \operatorname{COV}\left(d N^{+}, d N^{-}\right)=0 \\
\operatorname{COV}\left(d Z, d N^{+}\right)=0 ; \operatorname{COV}\left(d Z, d N^{-}\right)=0 \\
\operatorname{COV}(d W, d Z)=\rho d t
\end{gathered}
$$

$d N^{+}$se distribuye Poisson con parámetro de intensidad $\lambda^{+}$ $d N^{-}$se distribuye Poisson con parámetro de intensidad $\lambda^{-}$ 
$\nu^{+}$se distribuye Fréchet truncada con parámetros $\alpha^{+}, \mu^{+}$y $\sigma^{+}$, y con umbral $u^{+}$

$\nu^{-}$se distribuye Fréchet truncada con parámetros $\alpha^{-}, \mu^{-}$y $\sigma^{-}$, y con umbral $u^{-}$

Se observa que el modelo 1 es el clásico modelo de Merton-Black-Scholes. El modelo 2 incorpora volatilidad estocástica del tipo CIR (que asegura que la volatilidad sea siempre positiva).El modelo 3 es el más general de todos, pues los modelos del 1 y 2 son casos particulares del modelo 3 , en donde se han restringido los valores de ciertos parámetros a cero.

\section{Calibración y Resultados Econométricos}

1) Se calibrarán los 3 modelos con los datos de frecuencia diaria del indice de la BMV desde 1990 hasta 2006, y se obtendrá el valor de todos los parámetros necesarios para correr las simulaciones Montecarlo. La ventana de análisis es de aproximadamente 4 años (1000 datos), y se ajustarán los parámetros para todos los modelos moviendo esta ventana en el tiempo de manera trimestral. En total se obtuvieron 50 conjuntos de datos.

2) Una vez obtenidos los coeficientes que describen los procesos históricos en distintos momentos en el lapso 1990-2006, en 50 conjuntos de 1000 dias habiles (casi 4 años), se procederá a valuar un call y un put europeos a distintos plazos y con diferentes strikes, esto con cada uno de los 3 modelos que se ponen a prueba.

3) La valuación de los derivados se llevará a cabo mediante simulaciones Montecarlo, y se hará una simulación para cada conjunto de coeficientes obtenidos en el paso 1 y para cada modelo. Los detalles de la simulación Montecarlo se muestran posteriormente en este trabajo.

4) Se comparará el desempeño de los modelos comparando los precios teóricos obtenidos con los precios de derivados observados en el mercado mexicano de derivados estructurados.

5) Se mostrarán gráficas que muestran los precios obtenidos comparados con los teóricos de Black-Scholes.

Para la estimación econométrica se partirá la totalidad de observaciones (4105 observaciones en total) en subgrupos de 1000 (aproximadamente 4 años). La ventana de observaciones se moverá 62 ó 63 observaciones cada vez, de manera que se re-estimen los parámetros de las series de tiempo de manera aproximadamente trimestral, específicamente al último día hábil de cada trimestre. Esto se hace con el fin de estimar el valor teórico de los derivados con la información que se tendría disponible en el día en que se emite una nueva familia de derivados en el MexDer.

Las siguientes gráficas muestran al valor del índice de precios de la BMV, así como los log-rendimientos diarios: 
Gráfica 2.

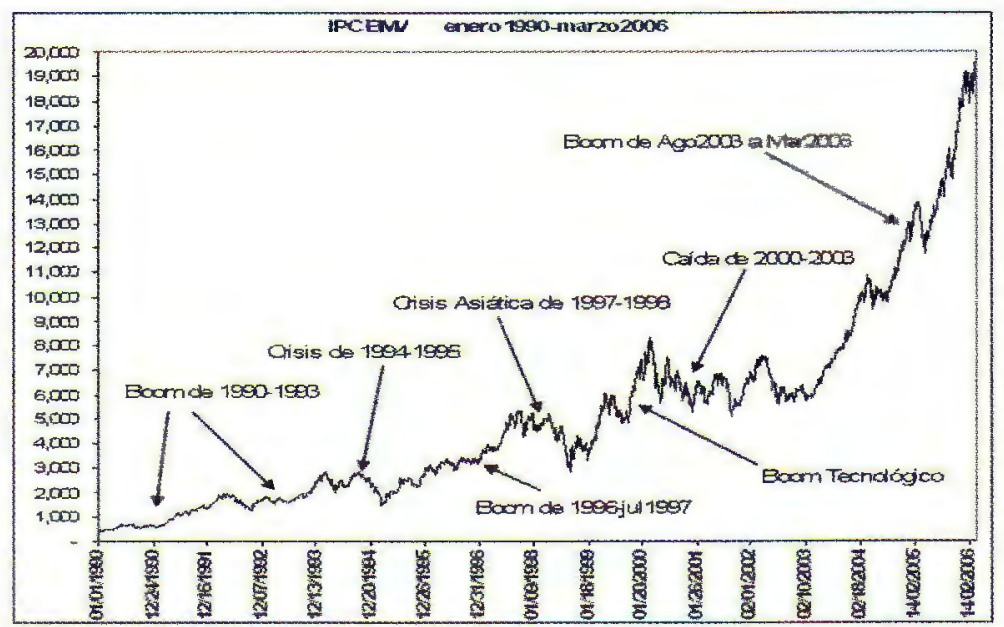

Gráfica 3.

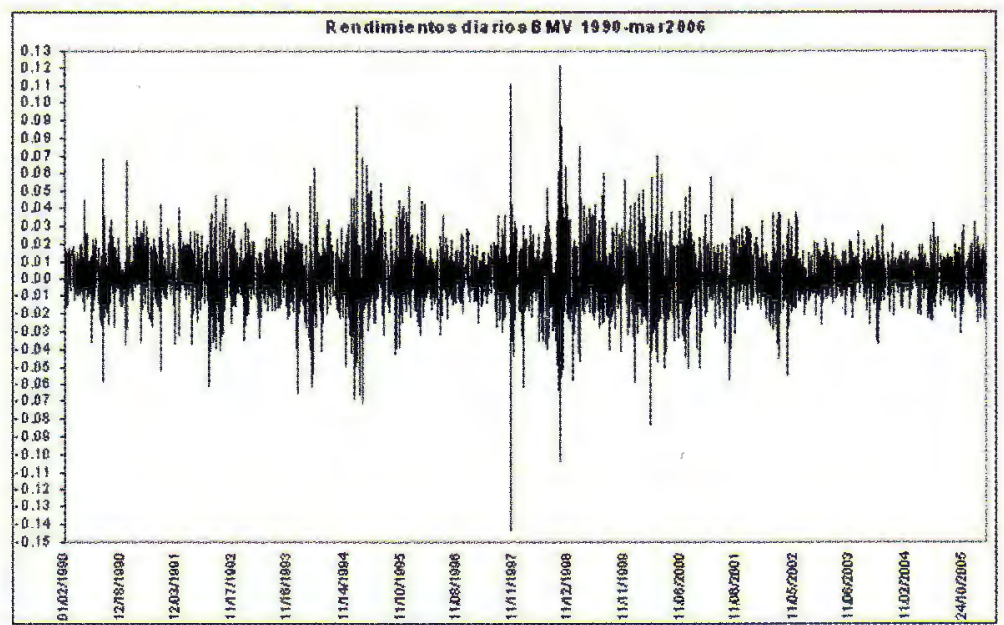

A continuación se muestran gráficas de los coeficientes J-B obtenidos para cada filtrado, tanto en la etapa 1 de la estimación (ajuste a los rendimientos observar dos) como en la etapa 2 (estimación de la ecuación de la volatilidad estocástica), donde se aprecia claramente que el filtro hacia arriba y hacia abajo es el que mayor normalidad presenta para los residuales de ambas etapas de estimación (se grafica el coeficiente J-B en escala logarítmica debido a las gigantescas diferencias observadas): 
Gráfica 4 .

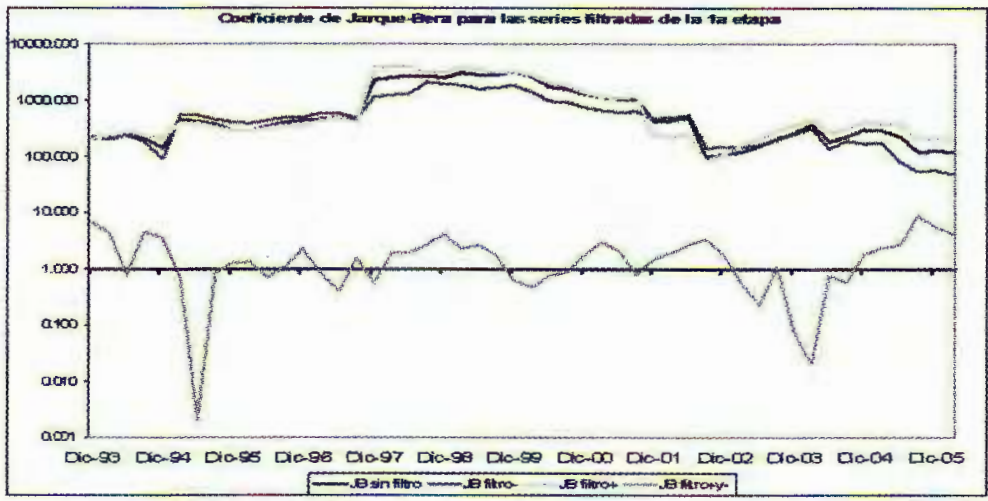

Gráfica 5.

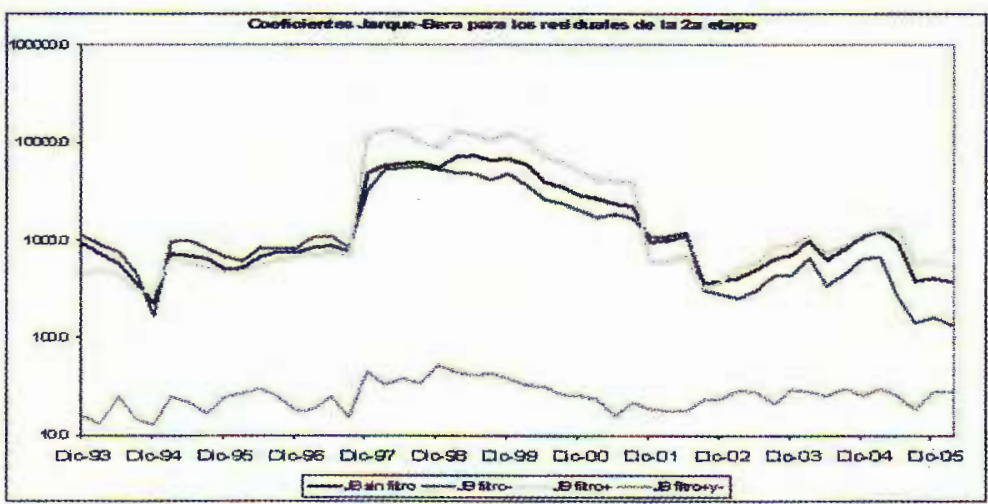

Gráfica 6 .

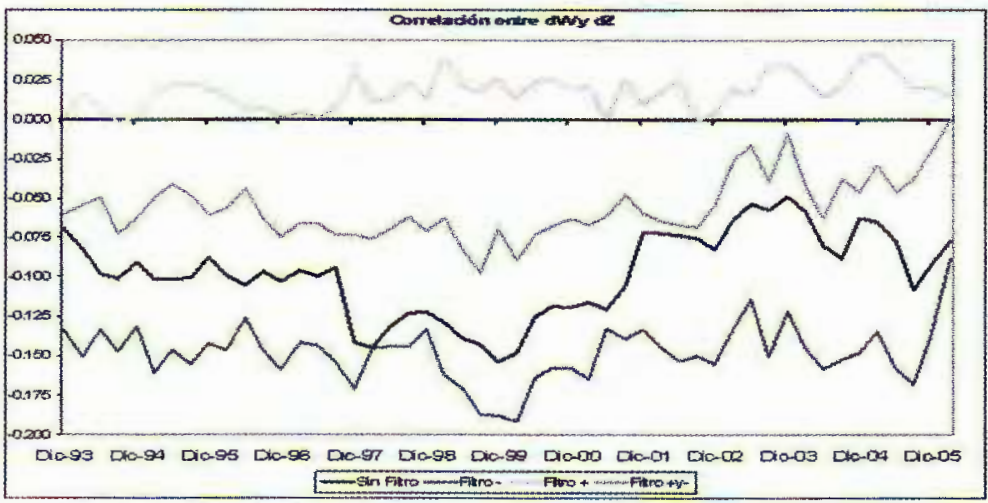

En la siguiente gráfica se observa que la correlación entre dW y dZ parece ser poco importante, pues generalmente se encuentra, en valor absoluto, por debajo de 0.2 . 


\section{Gráfica 7.}

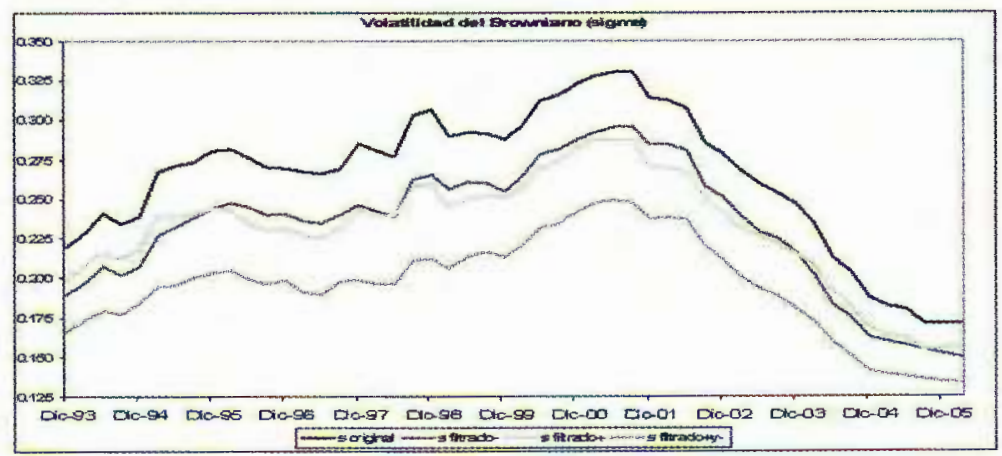

Es claro que de diciembre de 1993 a diciembre de 2001 la volatilidad $\sigma$ estimada fue creciendo. Esta fue la etapa de la burbuja financiera de las empresas tecnológicas. Esta burbuja explota a fines de 2001 y desde entonces hasta ahora la tendencia de 1000 observaciones (4 años) se encuentra en mínimos desde 1993. La volatilidad de la BMV se encuentra ahora en mínimos no vistos al menos desde 1993, y probablemente en mínimos no vistos hace décadas. La volatilidad de. la serie sin ningún filtro toca un máximo a fines de 2001 con un valor de más de 0.325 , y después cae hasta aproximadamente 0.20 en marzo de 2006 . La volatilidad de la serie con ambos filtros toma un valor máximo de cerca de 0.200 a fines de 2001, para bajar a cerca de 0.100 para marzo de 2006 .

Gráfica 8.

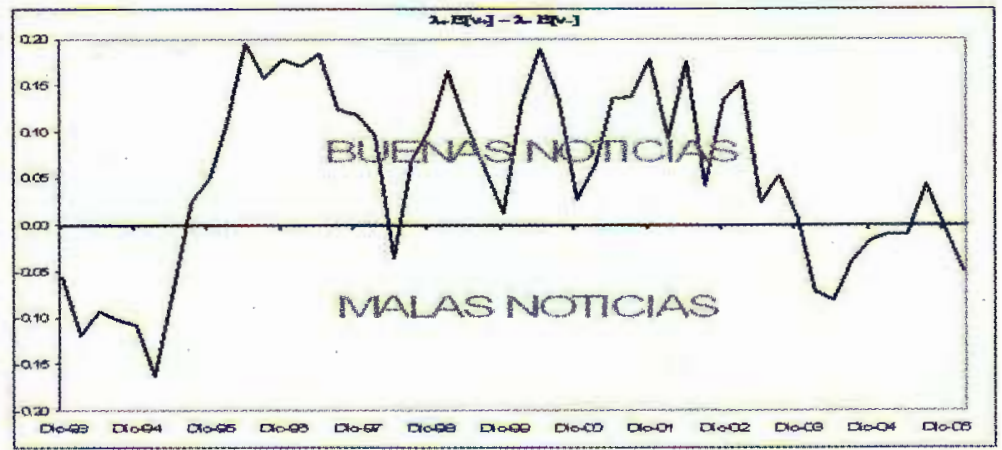

Se observa claramente que de diciembre de 1995 a diciembre de 2001 se vivió una temporada de continuo exceso de noticias positivas, mientras que de 1993 a 1995 se observa lo contrario. Para los 4 años hasta octubre del 2004 se observa una caída importante en este indicador, lo que es consistente con el panorama recesivo y de malas noticias que reinó desde el 2000 hasta finales del 2003. De fines de 2004 a marzo de 2006 este indicador muestra valores cercanos a cero. Se muestra ahora la gráfica de $\Psi$, que es la proporción de los saltos que fue asignado al movimiento browniano. Se aprecia que por lo general ronda entre el $0 \%$ y el $20 \%$ del total del salto. 
Gráfica 9.

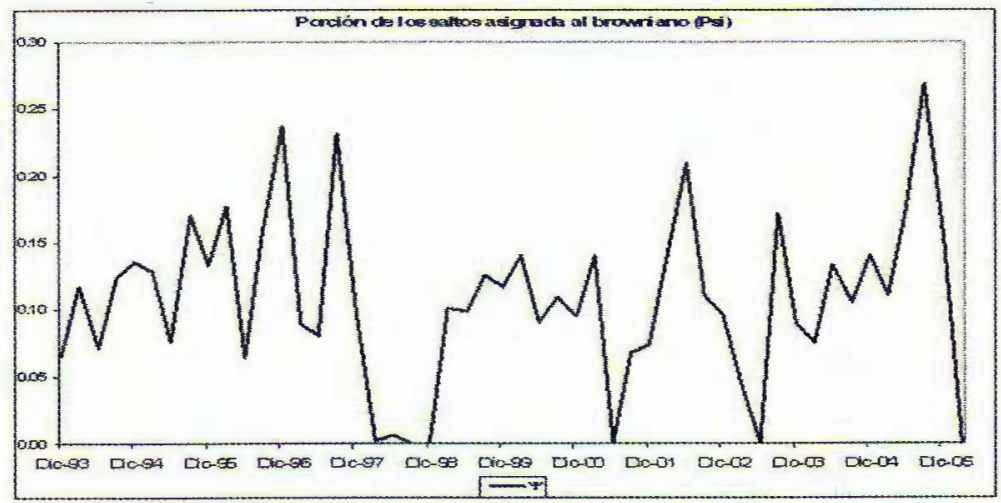

En resumen, durante la temporada de auge en la BMV 1999-2001 tanto la volatilidad, y la volatilidad de la volatilidad se incrementaron, y desde fines de 2001 hasta 2006 todas han disminuido sustancialmente. El mismo comportamiento se puede atribuir a los procesos de saltos. Esto tiene implicaciones importantes, pues entonces en temporadas de alta volatilidad del proceso browniano la importancia de los saltos es mayor que en temporadas de menor volatilidad (pues el producto de la intensidad de los saltos, $\lambda, y$ del valor esperado de los saltos, $E[\nu]$, es alto cuando la volatilidad del browniano es alta).

Esto implica entonces que el valor de los Calls o de los Puts europeos estarán muy subvaluados por la fórmula de Black-Scholes justo en los momentos en los que existe mayor riesgo (alta volatilidad). En otras palabras, valuar un Call o un Put Europeos a la Black-Scholes en momentos de alta volatilidad puede ser similar a hacer un suicidio financiero si lo que se busca es limitar el VaR de un portafolio de acciones que imite al indice de la BMV. Esto es importante para el emisor o poseedor actual de estos productos derivados, pues si valúa su VaR asumiendo que el modelo de Black-Scholes es correcto subestimará o sobreestimará consistentemente el VaR.

Otra implicación importante para las cámaras de compensación de derivados (y más que para ellos, para el regulador de dichos mercados) es que deberá replantearse integralmente la manera de medir los márgenes que deberán manejar para poder operar con el mínimo riesgo.

\section{Resultados de la estimación de los precios de Calls y Puts Euro- peos}

Se muestran a continuación los precios teóricos obtenidos para los Calls y los Puts Europeos. S[olo se muestran las gráficas para valores de $\mathrm{K}$ (el strike) igual al precio inicial del subyacente. Se muestran primero los valores obtenidos para los calls y después los valores obtenidos para los puts. Se muestran los resultados para maduraciones de $\mathrm{T}=0.25$ y $\mathrm{T}=5$ años. La línea punteada es el modelo de Black-Scholes, la rayada es el modelo con volatilidad estocástica tipo CIR y la sólida es el modelo que incluye saltos asimétricos: 
Gráficas obtenidas para los Calls:

Gráfica 10.

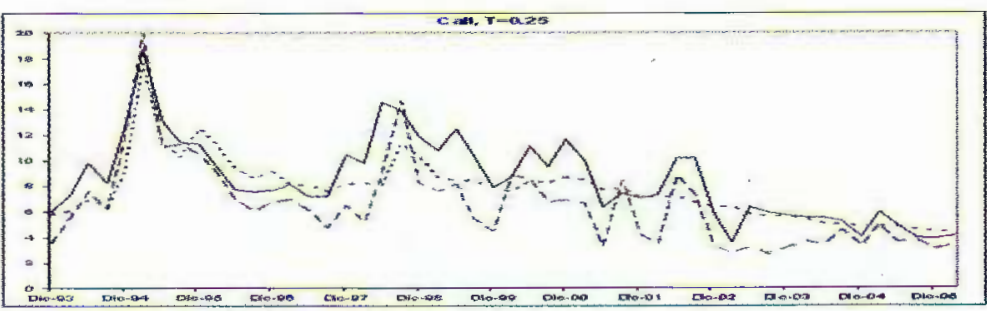

Gráfica 11.

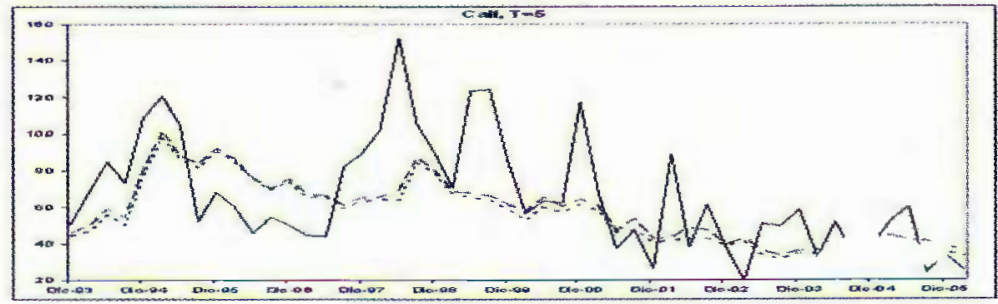

Gráficas obtenidas para los Puts:

Gráfica 12.

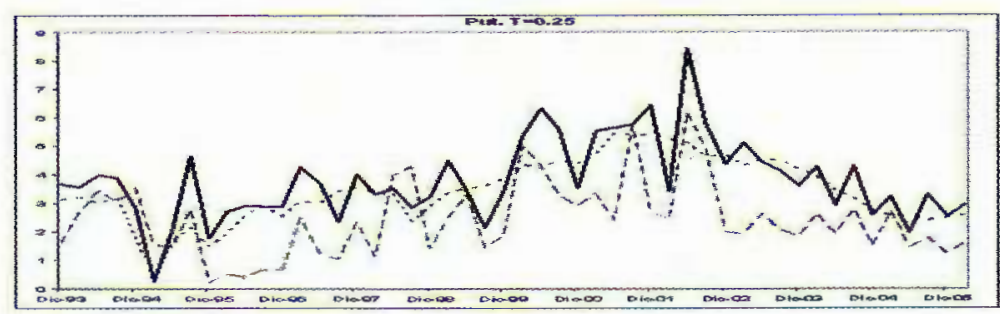

Gráfica 13.

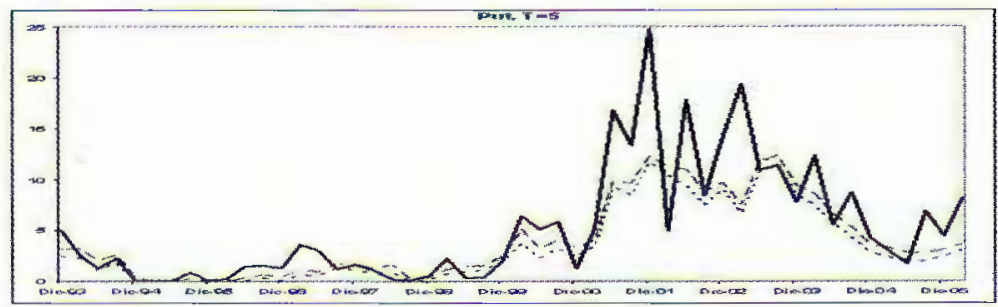


De estas gráficas se deduce inmediatamente que para plazos largos el modelo con volatilidad estocástica muestra siempre valores mayores que el de BlackScholes, consistente con los hallazgos de otros autores como Bates (1996), Ball (1994) y Hull-White (1987). También se observa que el modelo que incluye brincos permite precios por debajo de los obtenidos por B-S, y que muestra mayor varianza que el resto de los modelos. Destaca también que para fines del 2005 el modelo con saltos prevee precios por debajo de los observados por B-S, consistente con un mercado que espera un movimiento bajista en el futuro.De las gráficas de los Puts se deduce básicamente lo mismo que para los calls. En períodos de alta volatilidad y mercados bear el modelo predice precios por debajo de los obtenidos por B-S. Este comportamiento no puede ser reproducido por el modelo con volatilidad estocástica, pues la volatilidad es alta en esos momentos, lo que debería implicar un precio mayor al de Black-Scholes. Para Puts y Calls con maduración corta (digamos 3 meses) el efecto del valor inicial de la volatilidad, así como los saltos, parecen tener importancias similares. Esto implica que dichos instrumentos deben tomar ambos efectos en cuenta para valuar correctamente un derivado. Este tipo de instrumentos son típicos en el MexDer.

Se muestran ahora los "price smiles" que se obtienen para tres distintos momentos en el tiempo tanto para Calls como para Puts. Las fechas se escogieron de manera tal que se exploren distintos momentos en el mercado. Por ejemplo, a fines de 1995 dominaba el pesimismo, y lo contrario ocurría en 2001. Se muestran también las gráficas para la fecha en que se terminó este trabajo. Las gráficas siguen el orden descrito anteriormente. Las gráficas representan el cociente del precio obtenido entre el precio Black-Scholes menos uno. La linea horizontal en el cero es representa entonces al modelo de Black-Scholes, la linea discontinua representa al modelo con sólo volatilidad estocástica y la línea sólida, al modelo :

Gráficas obtenidas para los Calls:

Gráfica 14.

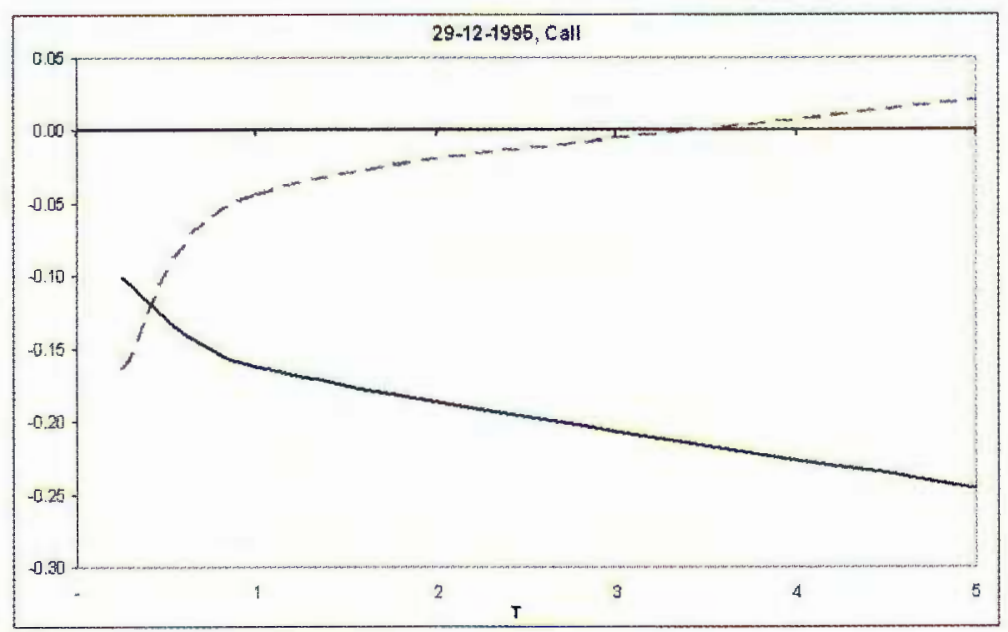


Gráfica 15.

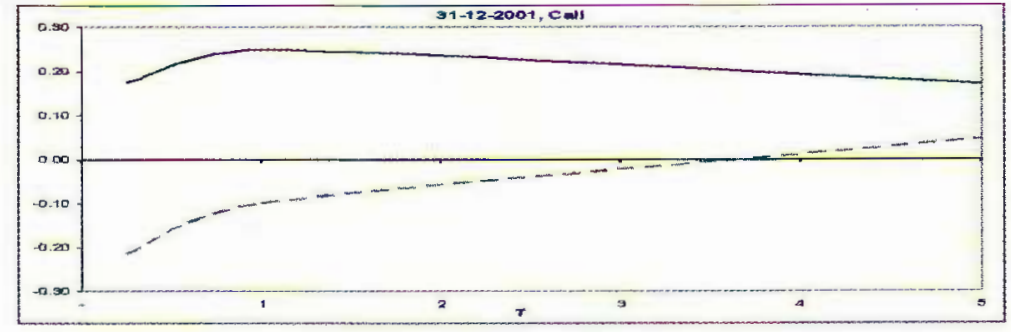

Gráfica 16.

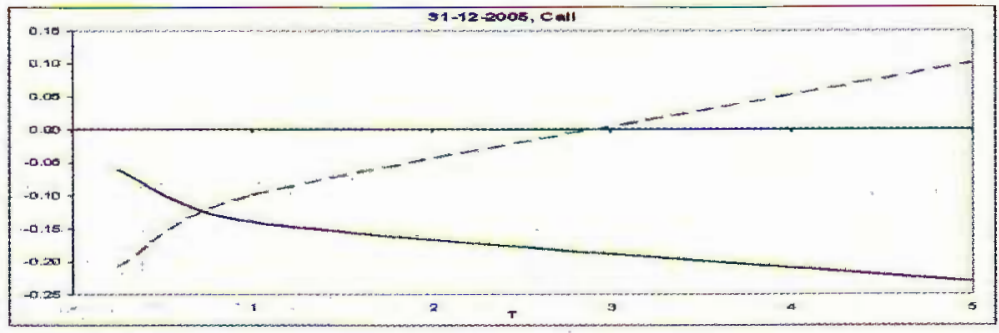

Gráficas obtenidas para los Puts:

Gráfica 17.

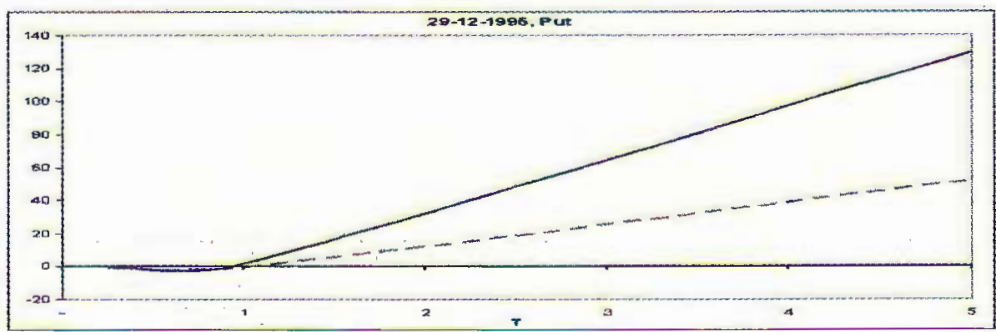

Gráfica 18.

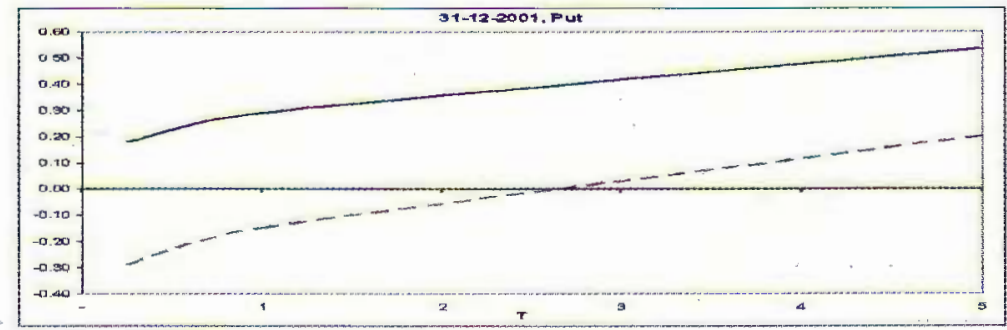


Gráfica 19.

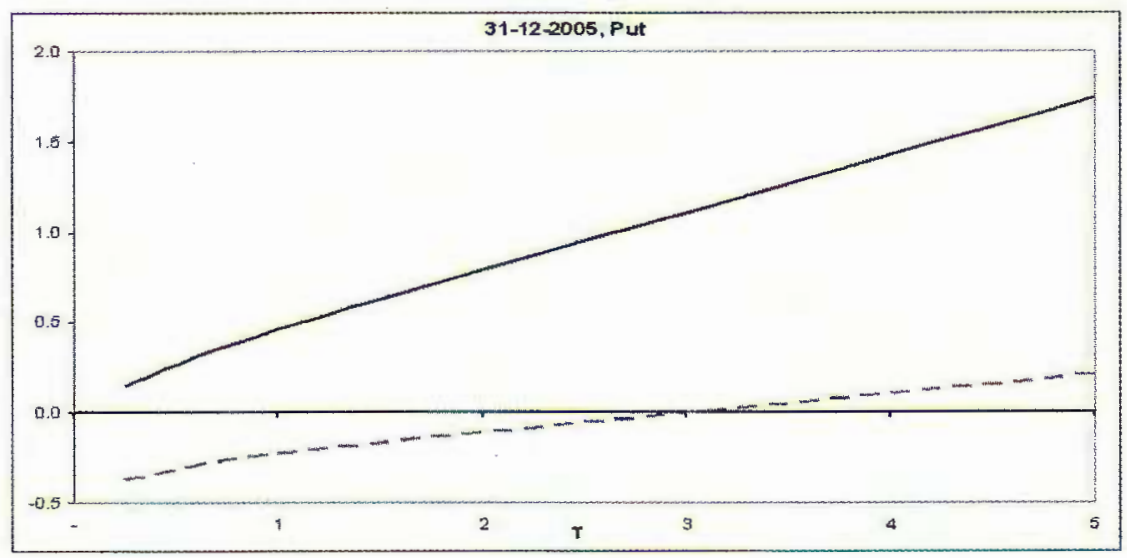

De las gráficas anteriores se observa que el modelo con sólo volatilidad estocástica suele valer más que el de Black-Scholes para maduraciones largas (5 años), y también que el modelo con volatilidad estocástica para maduraciones cortas tiende a valer menos que el de Black-Scholes. Esto se debe a que el período 2001-2005 ha observado una volatilidad descendente, por lo que los modelos con volatilidad estocástica son grandemente dependientes del calor inicial de la volatilidad que se tome. Para maduraciones a 3 meses (típicos del MexDer) el efecto de la volatilidad estocástica es importante, y también lo es el efecto de los saltos. Las siguientes gráficas muestran el comportamiento observado para la volatilidad implícita para Calls y Puts con maduración cercana a 1 trimestre y $\mathrm{K}$ cercana a $\mathrm{S}$ en el MexDer en el año 2004:

Gráfica 20.

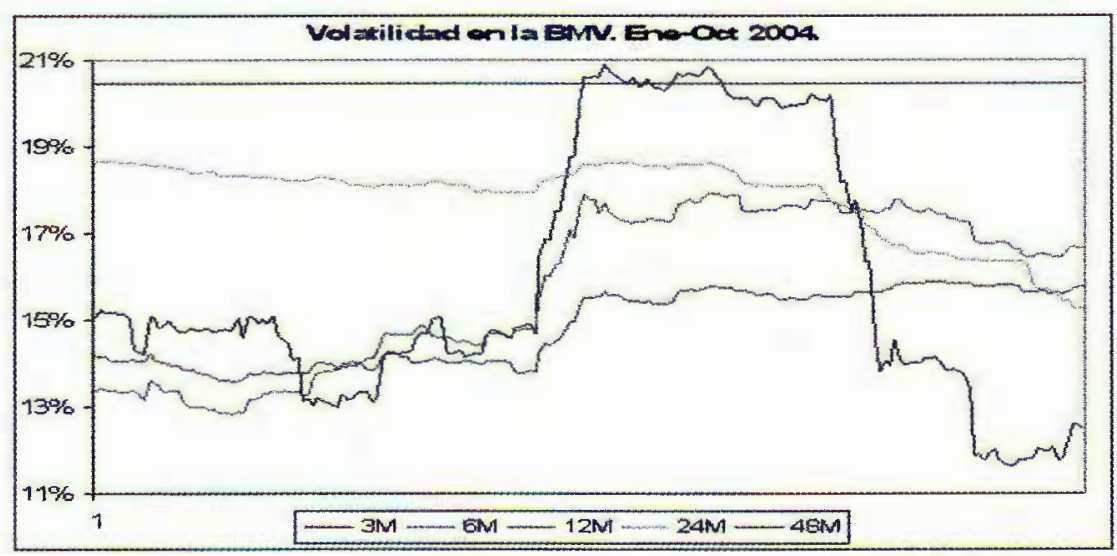


Gráfica 21.

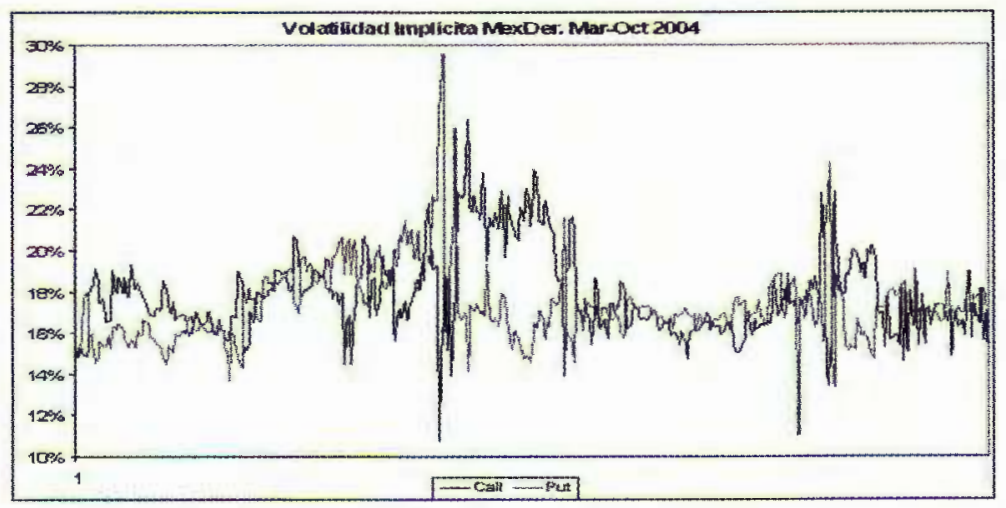

De las 2 gráficas arriba mostradas se advierten al menos dos cosas: 1) Las volatilidades implícitas de los Calls y de los Puts en el MexDer no son iguales en todo momento de tiempo. Esto es evidencia de que inclusive un modelo con volatilidad estocástica simétrico no podría explicar este comportamiento y que, por ello, un modelo con saltos asimétricos es candidato ideal; y 2) la BMV ha observado una baja significativa en la volatilidad durante el 2004-2006 y que en general la volatilidad implícita de los derivados en el MexDer es mayor que la volatilidad de la BMV cuando se mide para un período de 3 meses a 1 año, y que es generalmente menor cuando se mide a 4 años. Esto indica que, si el mercado piensa a la Black-Scholes, está midiendo la, volatilidad esperada para los siguientes 3 meses mediante una serie de tiempo a 2 años. A continuación se muestra una tabla donde se compara la distancia media ("MAPE" en inglés por Mean Absolute Prediction Error) entre las volatilidades de la BMV medidas con series de tiempo de distinta duración, y la volatilida implícita en el MexDer, donde se aprecia que la serie de tiempo a 2 años es la que mejor se ajusta a las volatilidades implícitas en el MexDer:

Tabla Comparativa 1

\begin{tabular}{|l|c|c|c|}
\hline & CALLS & PUTS & C-P \\
\hline MAPE $3 m$ & $3.157 \%$ & $3.068 \%$ & $0.069 \%$ \\
\hline MAPE 6m & $2.145 \%$ & $1.622 \%$ & $0.523 \%$ \\
\hline MAPE $12 m$ & $2.819 \%$ & $2.303 \%$ & $0.516 \%$ \\
\hline MAPE $24 m$ & $1.541 \%$ & $1.589 \%$ & $-0.049 \%$ \\
\hline MAPE $48 m$ & $4.086 \%$ & $4.601 \%$ & $-0.515 \%$ \\
\hline
\end{tabular}

\section{Conclusiones}

En general podemos concluir que la valuación de calls y puts europeos en la familia de modelos analizada se puede realizar de una manera relativamente rápida, y que es posible realizar los cálculos en una computadora casera actual. En promedio, la rutina programada en Fortran valúa los precios de los 3 
posibles derivados propuestos en esta familia de modelos (con una $\mathrm{T}=0.25$ ) en aproximadamente $1 / 2$ minuto.

Se concluye también que los modelos que permiten saltos hacia abajo y volatilidad estocástica permiten explicar comportamientos aparentemente extraños, como calls con precios teóricos menores a los obtenidos por BlackScholes. Además, el modelo 3 permite formas para el "volatility smile" que se adaptan bien a situaciones cambiantes del mercado, cosa que no sucede con modelos que sólo tienen brincos o sólo tienen volatilidad estocástica, como el modelo 2.

Además, se ratifica lo encontrado por otros autores (como Bates, 1996) en el sentido de que a mayor plazo de vencimiento $T$, mayor es el impacto de la volatilidad estocástica y menor es el efecto de los procesos de Poisson. Se ratifica también los hallazgos de que a plazos largos la volatilidad estocástica induce precios mayores que los observados por B-S, y que la diferencia en los precios es función creciente en T. El modelo propuesto en este trabajo permite explicar smiles tipo "frown" y smiles con tendencia decreciente en T, cosa que es imposible de lograr con un modelo exclusivamente con volatilidad estocástica tipo CIR.

Este trabajo propone una medida coherente de riesgo que resulta ser necesaria para poder valuar los derivados en cuestión. Una vez que se ajusta la distribución Fréchet a los máximos observados en el mundo real es posible evaluar rápidamente la medida coherente de riesgo que será incorporada en la valuación de los derivados. La posibilidad que tiene el modelo para permitir distribuciones de probabilidad de los logrendimientos asimétricas y con kurtosis y sesgo las hace ideales para modelar los activos financieros el mundo real.

Tal vez lo más interesante de los hallazgos de este trabajo es que a inicios del 2006 los precios teóricos obtenidos por los distintos modelos difieren mucho menos que en temporadas pasadas. Esto parece indicar que la fdp de los logrendimientos parece ser cada vez más normal.

Por otro lado, a inicios del 2006 se obtienen resultados interesantes al comparar los modelos 2 y 3 con el modelo $\mathrm{B}-\mathrm{S}$ para $\mathrm{T}=0.25$. El modelo 2 predice precios (especialmente de puts) más bajos que B-S. Esto se debe a la baja volatilidad que se observa últimamente relativa a la observada en los últimos 4 años. Por otro lado, el modelo 3 muestra precios de puts mayores que los propuestos por B-S. En 2004 los modelos con saltos tendían a observar precios de calls mayores a B-S, mientras que los modelos con sólo volatilidad estocástica tendían a observar precios menores que los de B-S. En 2006 se observa que un call tipo modelo 3 debería tener un valor menor que el de B-S, al igual que el modelo con volatilidad estocástica. Parece ser que los saltos hacia abajo son más intensos que los saltos hacia arriba, a pesar de que la volatilidad es baja en estos momentos (marzo 2006). Dados los altos niveles que observa el índice de la BMV en estos momentos y la tendencia a observar saltos habia abajo más intensos que hacia arriba podría ser señal de que es posible que inicie pronto un ciclo descendente en el índice ( $\mathrm{y}$ por ello los puts en el modelo 3 predicen precios mayores que B-S, y los calls precios menores a B-S).

Otra implicación importante de este trabajo es que hace inoperate al modelo clásico de las "betas" del CAPM, pues se muestra que la volatilidad no 
es constante y que existen observaciones extremas de carácter asimétrico. Esto es consistente con lo propuesto por Braun, Nelson y Sunier (Good News, Bad News, Volatility, and Betas), por Kim, M. e Ismail B. (An accounting analysis of the risk-return relationship in bull and bear markets) y Brown (Autocorrelation, Market Imperfections, and the CAPM).

Otro hallazgo interesante de este trabajo es que parece ser que la correlación entre el proceso browniano del subyacente y el de la volatilidad es pequeña. Esto nos permite decir que no hay grandes diferencias entre valuar un derivado tomando en cuenta dicha correlación o asumiendo que no existe tal correlación. Una correlación de cero hace aún más fácil y rápida la valuación numérica de los derivados, y parece ser que no es una mala aproximación. Esto es importante desde el punto de vista de parsimonia. Sin embargo, en momentos de alta volatilidad todo indica que la correlación tiende a crecer y en dichos momentos será mejor utilizar al modelo que incorpora la correlación.

En el 2004 inició el ciclo alcista en las tasas de interés en México y en muchos otros paises. Tal vez este hecho influya en la tendencia observada en la volatilidad, y, de ser cierto, habría que incorporar la correlación entre la tasa de interés y la volatilidad del índice de la BMV para valuar aún más correctamente los derivados. Este efecto de correlación no se toma en cuenta en este artículo, y puede ser un buen tema de investigación futura.

\section{Bibliografía}

Artzner, P., Delbaen, F., Eber, JM, Heath, D., (1999). Coherent measures of risk, Mathematical Finance, 9 (3), pp. 203-228.

Bakshi, G., Cao, C., Chen, Z. (1997), Empirical Performance of Alternative Option Pricing Models, The Journal of Finance, vol 52, no 5, pp. 2003-2049.

Ball, C., Roma, A. (1994), Stochastic Volatility Option Pricing, The Journal of Financial and Quantitative Analysis, Vol. 29, No. 4, pp. 589-607.

Bates, D. (1990), The Crash of '87: Was it expected? The evidence from Options Markets, The Journal of Finance, vol 46, no 3, pp. 1009-1044

Bates, D. (1996), Jumps and Stochastic Volatility: exchange rate processes Implicit in Deutsche Mark Options, Review of Financial Studies, vol 9, no 1, pp. 69-107.

Bera, A., Jarque, C. (1981), Efficient tests for Normality, Heteroscedasticity, and serial independence of regression residuals, Economics Letters, vol 7, pp. 313-318.

Black, F., Scholes, M. (1973), The Pricing of Options and Corporate Liabilities, Journal of Political Economy, vol 81, pp. 637-654

Black, F., Scholes, M. (1971), The Valuation of Option Contracts and a Test of Market Efficiency, The Journal of Finance, Vol. 27, No. 2, Papers and Proceedings of the Thirtieth Annual Meeting of the American Finance Association, New Orleans, Louisiana, December 27-29, pp. 399-417.

Bollerslev, T. (1986), Generalized Autoregresive Conditional Heteroscedasticity, Journal of Econometrics, vol 31, pp. 307-327.

Braun, P., Nelson, D., Sunier A. (1995), Good News, Bad News, Volatility, and Betas, The Journal of Finance, vol 50, no 5, pp. 1575-1603.

Brown S. (1979), Autocorrelation, Market Imperfections, and the CAPM, The Journal of Financial and Quantitative Analysis, vol 14, No 5, pp. 1027-1034.

Brown, T., (1984), Poisson Approximations and the Definition of the Poisson Process, The American Mathematical Monthly, vol 91, no 2, pp. 116-123.

Cox, J., Ingersoll, J., Ross, S. (1985), A Theory of the Term Structure of Interest Rates, Econometrica, Vol. 53, No. 2, pp. 385-408 
Cox, J., Ingersoll, J., Ross, S. (1985), An Intertemporal General Equilibrium Model of Asset Prices, Econometrica, Vol. 53, No. 2, pp. 363-384.

Duan, J., Simonato, J. (1998), Empirical Martingale Simulation for Asset Prices, Management Science, Vol. 44, No. 9, pp. 1218-1233.

Eberlein, E., Keller, U., Prause, K. (1998), New Insights into Smile, Mispricing, and Value at Risk: The Hyperbolic Model, The Journal of Business, Vol. 71, No. 3, pp. 371-405.

Engle, R., Ng, V. (1993), Measuring and Testing the Impact of News on Volatility, The Journal of Finance, Vol. 48, No. 5, pp. 1749-1778 .

Engle, R. (1982), Autoregresive Conditional Heteroscedasticity with estimates of the variance of the United Kingdom inflations, Econometrica, vol 50, pp. 987-1008.

Engle R., Lilien D., Robins R. (1987), Estimating time-varying risk premia in the term structure: The ARCH-M model, Econometrica, vol 55, No 2, pp. 391-407.

Figlewski, Stephen, Options Arbitrage in Imperfect Markets, The Journal of Finance, vol 44, no $5,1989,1289-1311$.

Fisher, L., Karnin, J. (1985), Forecasting systematic risk: Estimates of "raw" Beta that take account of the tendency of Beta to change and the heteroskedasticity of residual returns, The Journal of Financial and Quantitative Analysis, vol 20, No 2, pp. 127-149.

Geske R., Roll R. (1984), On valuing american call options with the Black-Scholes European Formula, The Journal of Finance, vol 39, No 2, pp. 443-455

Geske, R., Roll, R., Shastri, K. (1983), Over-the-Counter Option Market Dividend Protection and "Biases" in the Black-Scholes Model: A Note, The Journal of Finance, vol 38, no 4, pp. 1271-1277.

Heston, S., Nandi S. (2000), A Closed-Form GARCH Option Valuation Model, The Review of Financial Studies, vol 13, no 3, pp. 585-625.

Heston, S. (1993), A Closed-Form Solution for Options with Stochastic Volatility with Applications to Bond and Currency Markets, Review of Financial Studies, vol 6, no 2, pp. $327-343$.

Hilliard, J..; Reis, J. (1998), Valuation of Commodity Futures and Options Under Stochastic Convenience Yields, Interest Rates, and Jump Diffusions in the Spot, The Journal of Financial and Quantitative Analysis, Vol. 33, No. 1, pp. 61-86.

Hull, J., White, A. (1987), The Pricing of Options on Assets with Stochastic Volatilities, The Journal of Finance, vol 42, no 2, pp. 281-300.

Kim, M., Ismail B. (1998), An accounting analysis of the risk-return relationship in bull and bear markets, Review of Financial Economics, vol 7, No 2, pp, 173-182.

Kim, M., Oh, Y., Brooks, R. (1994), Are Jumps in Stock Returns Diversifiable? Evidence and implications for Option Pricing, The Journal of Financial and Quantitative Analysis, vol 29, no 4, pp. 609-631.

Markowitz, H. (1952), Portfolio Selection, The Journal of Finance, Vol. 7, No. 1 (Mar., 1952), 77-91.

Merton, R. (1976), Option Pricing when Underlying Stock Returns are Discontinuous, Journal of Financial Economics, vol 3, pp. 125-144.

Naik, V. (1993), Option Valuation and Hedging Strategies with Jumps in the Volatility of Asset Returns, The Journal of Finance, vol 48, no 5, pp. 1969-1984.

Page, F., Sanders, A. (1986), A General Derivation of the Jump Process Option Pricing Formula, The Journal of Financial and Quantitative Analysis, vol 21, no 4, pp. 437446.

Rabemananjara, R. (1993), Threshold ARCH models and asymetries in volatility, Journal of Applied Econometrics, vol 8, pp. 31-49.

Ritchken, P., Trevor, R. (1999), Pricing Options under Generalized GARCH and Stochastic Volatility Processes, The Journal of Finance, Vol. 54, No. 1, pp. 377-402.

Roll, R. (1988), $R^{2}$, The Journal of Finance, vol 43, No 3, Papers and proceedings of the forty-seventh annual meeting of the American Finance Association, Chicago, Illinois, pp. 541-566.

Turnovsky, S. (1993), Macroeconomic Policies, Growth, and Welfare in a Stochastic Economy, International Economic Review, vol 34, pp. 953-981. 
Turnovsky, S., Benavie, A., Grinols, E. (1996), Adjustment Costs and Investment in a Stochastic Equilibrium Macromodel Journal of Monetary Economics, 38, pp. 77-100.

Amemiya, Takeshi, Advanced Econometrics, Harvard University Press, 1996.

Boyarchenko, Svetlana I., Levendorskii, Sergei Z., Non-Gaussian Merton-Black-Scholes Theory, World Scientific, 2002.

Chiang, Alpha C., Elements of Dynamic Optimization, Waveland Press Inc, 2000.

Dempster, Michael A.H., Pliska, Stanley R, Mathematics of Derivatives Securities, Cambridge University Press, 1997.

Embrechts, Paul, Klüppelberg, Claudia, Mikosch, Thomas, Modelling extremal events for insurance and finance, Springer, 1999.

Goldberg, David E., Genetic Algorithms in Search, Optimization and Machine Learning, Addison Wesley, 1999.

Greene, William H., Econometric Analysis, 3a edición, Prentice Hall, 1997.

Handscomb, D.C., Methods of Numerical Approximation, Pergamon Press, 1965.

Hull, John C., Options, Futures and other Derivatives, 5th edition, Prentice Hall, 2003.

Jaeckel, P., Monte Carlo methods in Finance, Wiley Finance, 2000.

Johnson, Neil F., Financial Market Complexity, Oxford University Press, 2003.

Johnston, Jack, DiNardo, John, Econometric Methods, Mc Graw Hill, 1997.

Levy, H., Levy, M., Solomon, S., Microscopic simulation of financial markets: from investor behaviour to market phenomena, Academic Press, 2000.

Merton, Robert, Continuous-Time Finance, Blackwell, 1992.

Michalewicz, Zbigniew, Genetic Algorithms + Data Structures = Evolution Programs, 3a edición, Springer, 1999.

Musiela, Marek, Rutkowsky, Marek, Martingale Methods in Financial Modelling, Springer, 1998.

Willmott, Paul, Paul Willmott on Quantitative Finance, John Wiley \& Sons LTD, 2000. 